\title{
Full-hexahedral structured meshing for image-based computational vascular modeling.
}

Gianluca De Santis, Matthieu De Beule, Koen Van Canneyt, Patrick Segers, Pascal Verdonck, Benedict Verhegghe

\section{Address}

BioMMeda-IBiTech,

De Pintelaan 185,

B-9000 Gent,

Belgium

Phone: + $32(0) 93324621$

Corresponding author: gianluca.desantis@ugent.be

KEYWORDS: Image-based modeling, hexahedral mesh, centerline, patient-specific, Finite Element Analysis, Computational Fluid Dynamics, pyFormex 


\section{ABSTRACT}

Image-based computational modeling offers a virtual access to spatially and temporally high resolution flow and structural mechanical data in vivo. Due to inter-subject morphological variability, mesh generation represents a critical step in modeling the patient-specific geometry and is usually performed using unstructured tetrahedral meshing algorithms. Although hexahedral structured meshes are known to provide higher accuracy and reduce the computational costs both for Finite Element Analysis and Computational Fluid Dynamics, their application in computational cardiovascular studies is challenging due to the complex 3D and branching topology of vascular territories. In this study, we propose a robust procedure for structured mesh generation, tailoring the mesh structure to the subject-specific vessel topology. The proposed methodology is based on centerline-based synthetic descriptors (i.e. centerlines, radii and centerlines' normals) which are used to solve the meshing problem following a bottom-up approach. First, topologically equivalent block-structures are placed inside and outside the lumen domain. Then, a projection operation is performed, returning a parametric volume mesh which fits the original triangulated model with sub-micrometric accuracy. Additionally, a three-layered arterial wall (resembling the intima, media and adventitia) is artificially generated, with the possibility of setting variable thickness (e.g. proximal-to-distal tapering) and material anisotropy (e.g. position-dependent collagen-fibers' orientation). This new meshing procedure, implemented using open-source software packages only, is demonstrated on two challenging human cases, being an aortic arch and an abdominal aortic aneurysm. High-quality meshes are generated in both cases, according to shape-quality metrics. By increasing the computation accuracy, the developed meshing tool has the potential to further add "confidence" to the use of computational methods in vascular applications. 


\section{INTRODUCTION}

Previous research has shown that blood flow and wall stress patterns are etiologically associated with patho-physiological conditions, such as initiation of focal atherosclerotic manifestations (e.g. different flow fields can initiate an atheroprotective or an atheroprone endothelium phenotype), and may lead to acute adverse events (e.g. rupture of an aneurysm, stroke, infarction). While in-vivo imaging is part of clinical routine for diagnosis and treatment of atherosclerotic lesions, direct access to in-vivo mechanical data (using ultrasound imaging (US) $[1,2]$ and Phase Contrast Magnetic Resonance Imaging (PC-MRI) [2, 3]) offers limited spatial and temporal resolution. Image-based analysis is an indirect approach to compute accurate spatial and temporal data in vivo by modeling the physical system of interest as a set of partial differential equations, in which the domain is taken from medical images (Computer Tomography (CT), MRI, US) and boundary conditions are measured and/or assumed from physiological data [4]. Among the series of steps that need to be performed from segmentation to post-processing, mesh generation is critical, mainly because the geometry is not built in a bottomup process, like in Computer-Aided Design (CAD) applications for manufacturing industry, but appears all together in its complexity after segmentation [5, 6]. A medical image consists of a 3D array of grayscale intensities provided by clinical scanners in DICOM format (each cell of the array represents a specific volume of the patient-specific anatomy). With the segmentation process the geometry of the vascular segments of interest is extracted from the medical image and usually stored as a set of triangles in STL (stereolithography) format [6]. Owing to the geometrical complexity of the patient-specific domain defined by the STL surface, automatic meshing schemes using tetrahedral elements have been widely preferred for image-based analysis, both in Computational Fluid Dynamics (CFD) and Finite Element Analysis (FEA) 
applications, while structured hexahedral meshes have been rarely adopted or limited to simple geometries [7-11].

Recently, we have compared the performance of unstructured and structured meshes in solving the Navier-Stokes equations in a left coronary artery. As expected, tetrahedral (unstructured) meshes needed much higher resolution than structured meshes to reach mesh independency, with higher computational costs (computational time and memory). Interestingly, the wall shear stress did plateau with progressive refinement of structured meshes but not when unstructured meshes were used [12]. Such differences are also reported in other studies and are attributed to the high numerical diffusion error associated with unstructured meshes [13-15].

In Finite Element applications, it is well known that unstructured meshes (tetrahedrons, wedge elements) can produce acceptable displacement results but generally do not give accurate results for stress because they are very stiff shapes (poor bending and near incompressibility behaviour) $[16,17]$. A comparative study between different element types in FEA of blood vessels is not yet available; nonetheless, applications to human femur loading have shown that hexahedral meshes should be preferred over unstructured meshes (included quadratic tetrahedrons) in order to improve mesh independency [18].

In this paper, we present a robust procedure to generate structured and conformal meshes from triangulated surface models, suitable for CFD, FEA and Fluid Structure Interaction (FSI) investigation of vascular districts. A demonstration is given for two human cases of interest: an aortic arch and an abdominal aortic aneurysm (AAA). 


\section{MATERIALS AND METHODS}

A procedure for mesh generation of image-based vascular models has been implemented combining two open-source packages, the Vascular Modeling Toolkit (VMTK; a software for 3D reconstruction and geometric analysis of image-based modeling of blood vessels

[www.vmtk.org]) and pyFormex (a software for generating, transforming and manipulating large geometrical models of 3D structures by sequences of mathematical operations

[www.pyformex.org]). The four-step procedure (subsections $2.2-2.5$ ), required to generate a volume mesh of the lumen and wall from a triangulated surface model, is illustrated in detail on a human aortic arch, imaged with angio-MRI, and then applied on an AAA, imaged with CT angiography.

\subsection{Image segmentation}

An ostensibly healthy aortic arch imaged with angio-MRI was segmented in VMTK, generating a triangulated surface model (Fig. 1 A). After clipping the surface, flow extensions were added in the direction of the centerlines in order to enable flow development and circular borders for the applications of the boundary conditions (Fig. 1 B) [6]. 


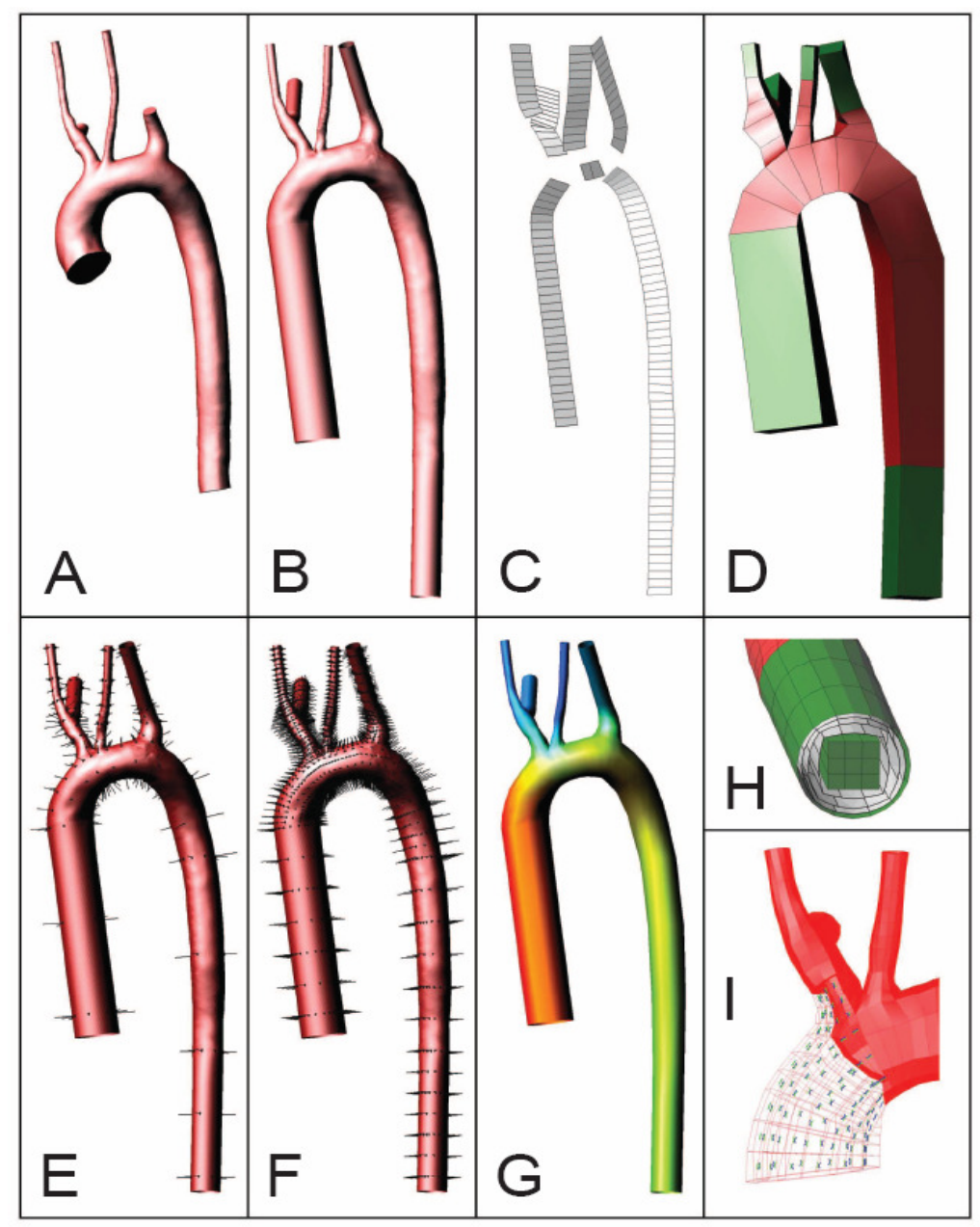

Fig. 1 - Steps required for the generation of a structured hexahedral mesh.

The triangulated surface model of the lumen (a) is given flow extensions (b) and processed to extract synthetic geometrical descriptors. In the figure, centerlines and parallel transport normals are shown as white rectangular panels twisting along the vessel branches. The orientation of the panels describes the binormals (c). Block-structures are built inside and outside the vessel, divided into different groups $(d, h)$ and projected on the lumen surface $(e, f)$. The local distance from centerline (i.e. a radius map $(g)$ ) can be used to recreate the arterial wall (i). Each element of the wall is featured with local triad axes, sufficient to characterize the arterial tissue anisotropy produced by helices of collagen-fibers. 


\subsection{Extraction of synthetic geometrical descriptors}

The aortic model with flow extension (STL format) was analyzed in VMTK in order to extract synthetic descriptors of the 3D structure: centerlines, radii and parallel transport normals. Centerlines describe the longitudinal evolution of the vessel branches (tube-like structures) and are determined as the paths on the Voronoi diagram that minimize the integral of the radius of maximal inscribed spheres along the path (i.e. the shortest paths in the radius metric). Each point of the centerline is then equipped with a local radius and triad axes (local reference system). The local axes are the abscissa (unit vector tangent to the centerline), the normal (a vector perpendicular to the centerline) and the binormal, which can be calculated as cross product of abscissa and normal. While the definition of the abscissa is straightforward, the normal in a set of curved and interconnected lines cannot be calculated using the local curvature (Frenet theory), as it would result undefined on straight segments and would experience abrupt changes in its orientation whenever the osculating plane changes (the osculating plane of a curve in a point is the plane that approximates the curve in the neighborhood of that point with second order contact). Following the implementation inside VMTK, the normal is defined as a parallel transport normal, being a unit vector perpendicular to the centerline which rotates on the osculating plane of an angle equal to the change of orientation of the centerline tangent while walking along the centerline. This iterative procedure defines an angular orientation along each centerline (i.e. circumferential position) without introducing artificial torsion. Each centerline's normal is then rigidly rotated to match the normals of the adjacent centerlines, using the first bifurcation as a landmark. This procedure is automatic and provides geometrical and topological descriptors of the vasculature (Fig. 1 C).

\subsection{Creation of block-structures}


Centerlines, maximal inscribed-sphere radii and parallel transport normals of each vessel branch are used within pyFormex to build two sets of topologically equivalent block-structures, externally and internally with respect to the vessel surface (Fig. 1 D). Such block-structures, which taper and twist in each vessel branch, result well shaped in geometrically simple regions, such as nearly straight branches, but are generally distorted in regions of branching (bifurcations or n-furcations), requiring manual correction for shape improvement. Inner and outer blockstructures are then converted into quadratic blocks and divided in different groups which may be used to separate the real aortic domain from the artificial flow extension in the post-processing phase (Fig. 1 D). More details are available in Appendix A.1.

\subsection{Projection of the block-structures and lumen mesh generation}

The quadrilateral surface surrounding the outer block-structure (outer surface) is projected towards the quadrilateral surface surrounding the inner blocks (inner surface) on the triangulated surface of the vessel, obtaining a quadrilateral mesh on the lumen surface (lumen surface (Fig. 1 H)). To decrease the differences between the triangulated model and the new quadrilateral mesh, a higher number of projection points can be set by splitting in an isotropic fashion all blocks before projecting (Fig. 1 E, F). The new quadrilateral mesh of the lumen surface can be connected to the topologically equivalent mesh of the inner surface to generate new (volume) block-structures.

These new blocks, which can be refined in the radial direction to have a near-wall finer mesh (Fig. 1 H), are added to the original inner block-structures, obtaining a quadratic hexahedral mesh of the vessel lumen. Such parametric mesh can then be converted into a linear mesh of desired resolution by isotropic refinement, suitable for CFD calculations. 


\subsection{Vessel wall model generation and meshing}

In most cases the entire wall surface is not available and needs to be reconstructed by either assigning a constant wall thickness or, more realistically, assigning a radius-dependent wall thickness (i.e. a percentage of local radius as distance from the centerline [19-21]) (Fig. 1 G). The wall surface can then be obtained displacing the lumen surface in the direction of the projection (from inner surface to outer surface) by a distance equal to the thickness (constant or radius-dependent). When both the lumen surface and wall surface are available, generating the quadrilateral surface mesh on both of them is trivial as it is sufficient to repeat twice the projection operation described in subsection 2.4, first using the lumen surface and then using the wall surface. In both cases, the inner and outer wall quadrilateral meshes have the same topology and can be directly connected into a volume mesh (by connecting two quadrilateral meshes with the same topology a hexahedral mesh is generated). Inside the wall several layers can be generated, for example, to resemble the three-layered vessel-wall structure (intima, media and adventitia), and in each layer hexahedral elements will be oriented according to the longitudinal, radial and circumferential direction (Fig. 1 I). Remarkably, if the surface of each layer is available from the segmentation or the local thickness of each layer is measured (for example using Intravascular Ultrasound Imaging), a mesh of the patient-specific layered wall can be easily generated by (1) repeating on each layer the projection operation described in subsection 2.4 to generate a series of quadrilateral surface meshes (from inner to outer) and then (2) connecting the quadrilateral surface meshes together to generate hexahedral meshes in each layer.

\subsection{Mesh quality inspection}


The quality of the mesh needs to be evaluated before using it for computational analysis. Among the metrics available as a measure of mesh quality, equi-angle skew and scaled Jacobian are the most common for CFD and FEA applications, respectively. The parameter equi-angle skew (Eq. 1) is a normalized measure of skewness ranging from 0.0 (best) to 1.0 (worst) and depends on the angle formed between the faces' edges of each cell in the mesh $(0.0$ corresponds to a perfectly equiangular cell) [12]:

equi-angle skew $=\max \left[\frac{\theta_{\max }-90}{90}, \frac{90-\theta_{\min }}{90}\right]$

[Eq. 1]

where $\theta_{\max }$ and $\theta_{\min }$ are the largest and smallest angles in the cell.

The Jacobian $J$ evaluated at a hexahedron's node $\mathrm{k}$ is the triple scalar product of the edges connected to that node $\left(e_{k 1}, e_{k 2}, e_{k 3}\right)$ and its module represents six times the local volume of the tetrahedron enclosed by the corresponding triad edges (Eq. 2). The scaled Jacobian of a hexahedron is the minimum Jacobian divided by the lengths of the 3 edge vectors (Eq. 3).

$J_{k}=e_{k 1} \cdot\left(e_{k 2} \times e_{k 3}\right) \quad[\mathrm{Eq} .2]$

scaled Jacobian $=\min \left[J_{k} /\left(e_{k 1} e_{k 2} e_{k 3}\right)\right]$, with $k=0,1 . .7$

[Eq. 3]

The scaled Jacobian can range from -1 (worst) to +1 (best) but elements with negative scaled Jacobian are not recommended for analysis [22]. 


\section{RESULTS}

A structured hexahedral mesh has been generated inside the lumen of a human aortic arch and an AAA (Fig. 1 A and Fig. 3 A), by combining a series of central block-structures with four series of lateral block-structures, as displayed in the cross-section of Fig. 2 C. In order to separate the aortic domain from the artificial extensions the elements of the aortic arch have been grouped in two sets (Fig. 2 A, B).

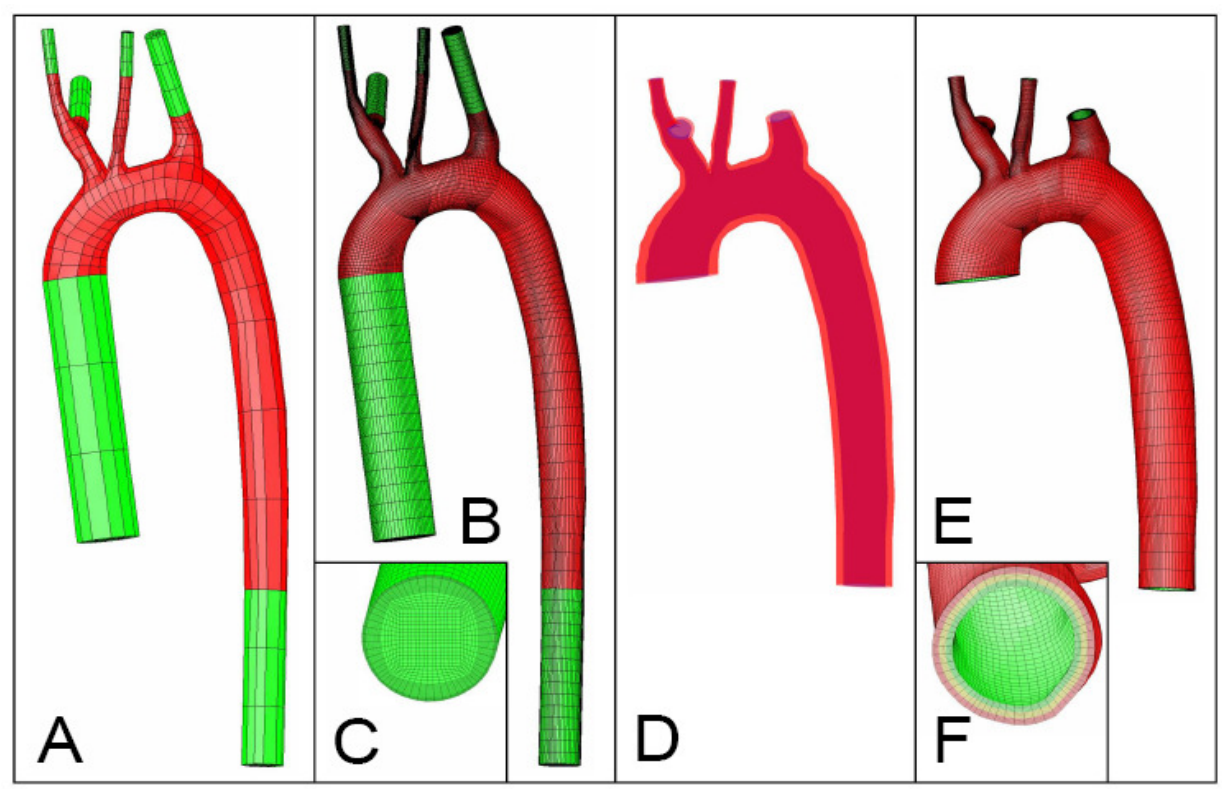

\section{Fig. 2 - Mesh of a human aortic arch.}

An initial mesh has been generated inside the lumen (a) and the wall (d, transparent view showing the tapering) of an aortic arch and then refined $(b, c, e, f)$. Inside the wall, three concentric layers have been defined, resembling the three-layered structure of the arterial tissue $(e, f)$. The number of cells (individual finite elements) is reported in Table 1.

The outer contour of the vessel wall was not available from the images and needed to be reconstructed from anatomical data. Distal-to-proximal tapering was taken into account when 
constructing the aortic-arch wall by assigning a thickness equal to a third of the local radius (this value was an arbitrary choice and did not influence the generality of the mesh generation procedure) (Fig. 2 D). The wall mesh in the aortic arch model has been partitioned into three layers resembling intima, media and adventitia (Fig. 2 F), without altering the longitudinal and the circumferential topology of the mesh, thus keeping a perfect conformal matching between the mesh of the inner wall surface and the mesh of the lumen surface (Fig 2 C, F). Each element was equipped with longitudinal (distal to proximal), circumferential (counterclockwise rotation around the centerline) and radial (inner to outer) directions, using the vectors connecting the centers of opposite faces of each hexahedron. Using this hexahedron-specific cylindrical coordinate system, the direction of counter-rotating helical collagen fibers inside the wall could be defined (as an example, Fig. 1 I shows the helices produced using an opening angle of 70 degrees from the circumferential vector on the plane normal to the radial direction [23]).

The wall of the AAA was reconstructed without layers and without taking into account the tapering. A constant thickness of $1 \mathrm{~mm}$ was assumed in order to eliminate the influence of the radius of the aneurysmatic lumen which would produce an abnormally thicker wall. (Fig. 3 B, C). 


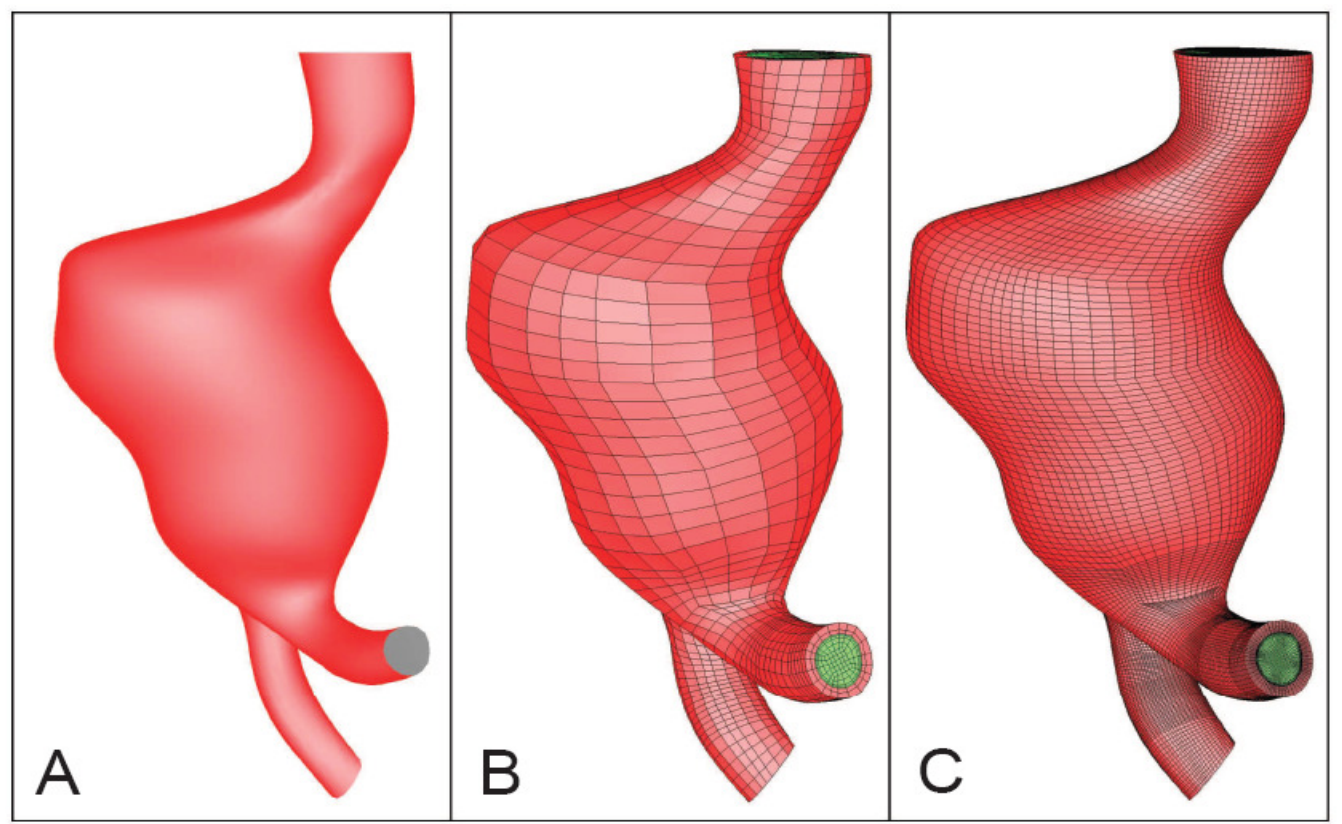

Fig. 3 - Mesh of human AAA.

The image-based lumen surface of an $A A A(a)$ has been used to generate a conformal volume mesh inside the lumen and the wall (lumen cells and wall cells) (b). A finer mesh (lumen cells and wall cells) has been obtained without altering the mesh topology, by refining each quadratic element (c). The number of cells is reported in Table 1.

The volume mesh inside the lumen was generated as combination of a mapping and a projection operation, in which block-structures were deformed to match the original surface model. The geometrical error, quantified as local distance between the surface of the meshed lumen and the original surface, was lower than one micron, thus within the numerical (round-off) error of the computation infrastructure, as reported in Table 1. 

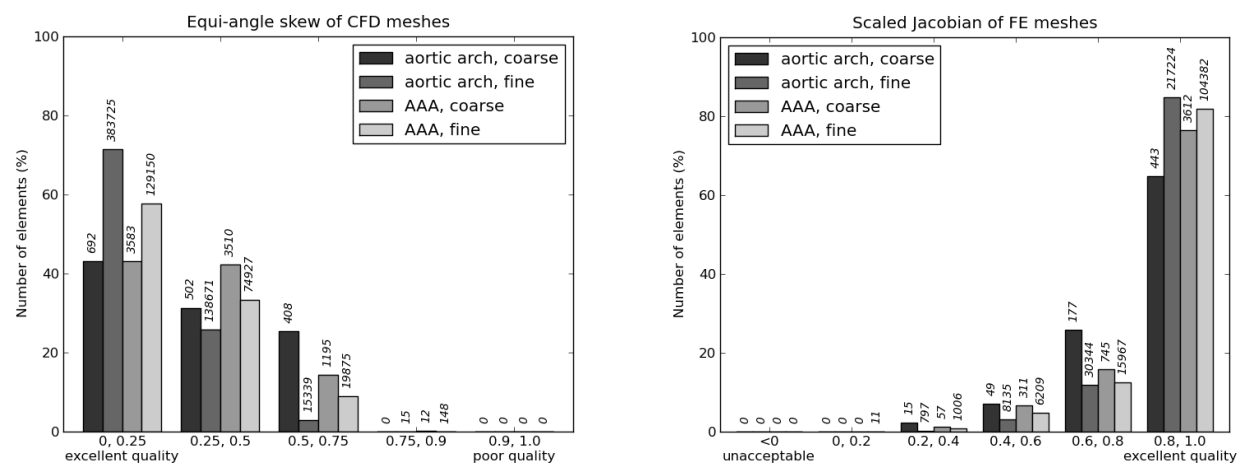

Fig. 4 - Mesh quality measure.

Equi-angle skew (a) and scaled Jacobian (b) distribution for the meshes of aortic arch and AAA (mesh features reported in Table 1).

The average equi-angle skew of the lumen meshes was below 0.4 (Fig. 4 A), which is optimal for CFD applications (0.32/0.19 for the coarser/finer aortic arch and 0.30/0.25 for the coarser/finer AAA) [7]. The average scaled Jacobian of the wall meshes was higher than 0.8 (Fig. 4 B), which is optimal for FEA applications (0.81/0.90 for the coarser/finer aortic arch and $0.86 / 0.89$ for the coarser/finer AAA) [22].

Initial mesh generation required 4 and 0.5 hours for the aortic arch and the AAA, respectively, and most of the time was spent to adjust the block-structures. As a comparison, the segmentation process required one day work for the aortic arch and few hours for the AAA. After the first mesh generation, the parametric nature of the structured mesh allowed remeshing without altering the mesh topology, performing the remeshing in a matter of minutes (Fig. 2 B, Fig. 3 C, Table 1). To increase the mesh density, the block-structures can be refined before projection or after projection (see Appendix A.2). In the former case, the finer mesh is expected to improve the surface representation while in the latter case the finer mesh does not corresponds to a more 
accurate representation of the geometry. Increasing the number of elements by two order of magnitudes (multiplying the number of elements by a factor 256 corresponds to shortening the elements' edges by a factor 8) by refinement after projection, preserved the geometrical accuracy below one micron (Table 1). To test whether a refinement before projection would compromise the mesh quality, we have generated an extra mesh of the aortic arch by increasing the quadratic patches from 900 to 4900 , thus increasing the number of projected points from 2768 to 14864 (generating 769986 and 301644 cells in the lumen and in the wall, respectively). The average and maximum value of Equi-angle skew did not change $(0.195 / 0.759$ and $0.188 / 0.763$ for the lumen reconstructed with 900 and 4900 patches, respectively) while the average and minimum value of scaled Jacobian was found 0.90/0.27 and 0.90/0.21 for the wall reconstructed with 900 and 4900 patches, respectively.

\begin{tabular}{|l|l|l|l|}
\hline \multicolumn{2}{|l|}{ Meshes } & Aortic arch & AAA \\
\hline \multirow{4}{*}{ Coarse } & Lumen (elements) & 1602 & 8300 \\
\cline { 2 - 4 } & Wall (elements) & 684 & 4725 \\
\cline { 2 - 4 } & Meshing time (h) & 4 & 0.5 \\
\cline { 2 - 4 } & Luminal-surface & mean 0.017 & mean 0.2 \\
\hline \multirow{3}{*}{ Fine } & accuracy (micron) & max 0.1 & max 0.7 \\
\cline { 2 - 4 } & Lumen (elements) & 537750 & 224100 \\
\cline { 2 - 4 } & Wall (elements) & 256500 & 127575 \\
\cline { 2 - 4 } & Time to remesh (s) & 132 & 92 \\
\hline
\end{tabular}

Table 1 - Mesh features in the aortic arch and AAA. 
Coarse and fine meshes have been generated by projecting the same number of quadratic patches, (900 for the aortic arch and 1575 for the AAA) and the accuracy of the luminal surface (the perpendicular distance between the local surface geometric representation and the nodes of the finite element mesh) remained below one micron. The initial meshes required extensive operator-intervention (up to four hours) while successive remeshing operation could be performed within three minutes. 


\section{DISCUSSION}

In this study the problem of structured hexahedral mesh generation for image-based computational analysis of vascular districts is solved using a novel procedure. First, a multi-block grid is fitted inside the vessel volume, at a distance from the lumen surface. Then, a series of layers are projected from the surface of the grid on the lumen surface, and, if needed, radiated outside the lumen to model the vessel wall. For a patient-specific geometry, block-structures are generated using synthetic geometrical descriptors, enabling mesh construction in a bottom-up approach.

In previous works, we have solved the problem of structured meshing in a patient-specific carotid artery bifurcation using a combination of surface slicing, spline reconstruction and mapping $[8$, 21]. Other authors have developed alternative methods for one single bifurcation or nearly-planar multiple bifurcations [7, 9-11, 14, 24]. However, when vascular districts appear non-planar, with centerlines evolving out of one single plane, the vessel twisting (twist angle, rotation of the centerline's normal around the centerline's tangent) needs to be taken into account for the generation of structured meshes. In the above mentioned nearly-planar cases, the twist angle could silently be taken as zero by watching the bifurcation along the bifurcation axis [25].

The construction of block-structures inside and outside the vessel is guided by the synthetic descriptors (centerlines, equipped with normals and radii), generating specific connecting patterns at branching locations. The surface of the block-structures is then radiated on the lumen surface, filling the entire volume with a structured grid. Projections along the surface normals are commonly used in mesh generation but are not suitable in regions of marked curvature, where negative-Jacobian elements (i.e. negative signed volumes) are produced. To overcome this 
limitation, each projecting ray is not oriented along the surface outward normal, but directed from a source point to a target point (the source points are the surface nodes of the inner blockstructures and the target points are the surface nodes of the outer block-structures). As a result, well shaped elements are generated not only in nearly-straight branches, but also in regions of branching (e.g. aortic arch) and marked curvature (e.g. AAA).

The new approach allows recreating the vessel wall (difficult to image, especially in branching regions) with a constant thickness or, more realistically, using the local vessel size as surrogate of thickness (thicker wall in larger vessels, generally located proximally). The source-to-target radiation operation avoids the risk of generating negative-Jacobian elements and ensures that the hexahedrons inherit the orientation of the original block-structures. Local orientation (longitudinal, circumferential and radial) allows defining material anisotropy, as, for example, counter-rotating helices of collagen fibers with layer-specific pitch angles [23]. Lumen and wall meshes are conformal (no hanging nodes and perfect edge/faces matching) and, if needed, can be couples (same surface mesh at interface), enabling a one-to-one correspondence of structural and flow data for FSI applications.

As an example, hexahedral meshes have been generated in a human aortic arch and an AAA, requiring hours for the initial mesh and minutes for successive refinements, obtained by remeshing original quadratic isoparametric models. The meshes reproduced the original surfaces with sub-micrometric accuracy and had high shape quality, independent of the resolution. Instead of refining after projecting, a finer mesh can also be obtained by refining the block-structures before projecting, thus increasing the number of projection rays. We tested the two remeshing options on the aortic arch but we did not find a significant difference neither in the surface 
accuracy nor in the mesh quality. This can be ascribed to the fact that the surface representation obtained with the coarse mesh was already adequate. In general, checking a posteriori the accuracy of the surface representation and the quality of the volume mesh is recommended before starting the computation. However, in case of a coarse STL model, attention needs to be paid when the resolution of the new mesh reaches the resolution of the original surface model, as aliasing effects can introduce spurious geometrical edges which, in turn, can generate artifacts in the computational analysis (e.g. stress concentration in case of FEA and disturbed flow in CFD). Improving the original STL model by repeating the segmentation or by a smoothing operation would avoid such effects.

To the authors' knowledge, this is the first successful attempt to generate lumen and wall structured meshes in large vascular districts. General-purpose hexahedral mesh generators have been developed (e.g. spatial twist continuum [26]) but applications to vascular districts are rare mainly because of the requirements needed in the vessel mesh (e.g. for the lumen, stretched elements in longitudinal direction and closely-layered elements in radial direction, following the expected velocity gradient; for the wall, closely-layered elements in radial direction and triad axes specification in each element to define material anisotropy). Current improvements are aiming at developing algorithms for local conformal refinement in hexahedral meshes and at meshing non-vascular patient-specific geometries (e.g. femur) and orthopedics devices (e.g. joint prosthesis). 


\section{REFERENCES}

1. Swillens, A., T. De Schryver, L. Lovstakken, H. Torp, and P. Segers, Assessment of Numerical Simulation Strategies for Ultrasonic Color Blood Flow Imaging, Based on a Computer and Experimental Model of the Carotid Artery. Annals of Biomedical Engineering, 2009. 37(11): p. 2188-2199.

2. Ponzini, R., M. Lemma, U. Morbiducci, F.M. Montevecchi, and A. Redaelli, Doppler derived quantitative flow estimate in coronary artery bypass graft: A computational multiscale model for the evaluation of the current clinical procedure. Medical Engineering \& Physics, 2008. 30(7): p. 809-816.

3. Morbiducci, U., R. Ponzini, G. Rizzo, M. Cadioli, A. Esposito, F. De Cobelli, A. Del Maschio, F.M. Montevecchi, and A. Redaelli, In Vivo Quantification of Helical Blood Flow in Human Aorta by Time-Resolved Three-Dimensional Cine Phase Contrast Magnetic Resonance Imaging. Annals of Biomedical Engineering, 2009. 37(3): p. 516531.

4. Taylor, C.A. and D.A. Steinman, Image-Based Modeling of Blood Flow and Vessel Wall Dynamics: Applications, Methods and Future Directions. Annals of Biomedical Engineering, 2010. 38(3): p. 1188-1203.

5. Svensson, J., R. Gardhagen, E. Heiberg, T. Ebbers, D. Loyd, T. Lanne, and M. Karlsson, Feasibility of patient specific aortic blood flow CFD simulation, in Medical Image Computing and Computer-Assisted Intervention - Miccai 2006, Pt 1, R. Larsen, M. Nielsen, and J. Sporring, Editors. 2006. p. 257-263.

6. Antiga, L., M. Piccinelli, L. Botti, B. Ene-Iordache, A. Remuzzi, and D. Steinman, An image-based modeling framework for patient-specific computational hemodynamics. Medical \& Biological Engineering \& Computing, 2008. 46(11): p. 1097-1112.

7. Antiga, L., B. Ene-Iordache, L. Caverni, G.P. Cornalba, and A. Remuzzi, Geometric reconstruction for computational mesh generation of arterial bifurcations from CT angiography. Computerized Medical Imaging and Graphics, 2002. 26(4): p. 227-235.

8. De Santis, G., M. De Beule, P. Segers, P. Verdonck, and B. Verhegghe, Patient-specific computational hemodynamics: generation of structured and conformal hexahedral meshes from triangulated surfaces of vascular bifurcations. Computer Methods in Biomechanics and Biomedical Engineering. in press, DOI 10.1080/10255842.2010.495066.

9. Longest, P.W. and C. Kleinstreuer, Numerical Simulation of wall shear stress conditions and platelet localization in realistic end-to-side arterial anastomoses. Transactions of the ASME. Journal of Biomechanical Engineering, 2003. 125(5): p. 671-81.

10. Longest, P.W. and C. Kleinstreuer, Particle-hemodynamics modeling of the distal end-toside femoral bypass: effects of graft caliber and graft-end cut. Medical Engineering \& Physics, 2003. 25(10): p. 843-858.

11. Longest, P.W., C. Kleinstreuer, and A. Deanda, Numerical simulation of wall shear stress and particle-based hemodynamic parameters in pre-cuffed and streamlined end-to-side anastomoses. Annals of Biomedical Engineering, 2005. 33(12): p. 1752-1766.

12. De Santis, G., P. Mortier, M. De Beule, P. Segers, P. Verdonck, and B. Verhegghe, Patient-specific computational fluid dynamics: structured mesh generation from coronary angiography. Medical \& Biological Engineering \& Computing, 2010. 48(4): p. 371-380.

13. Prakash, S. and C.R. Ethier, Requirements for mesh resolution in 3D computational hemodynamics. Journal of Biomechanical Engineering-Transactions of the Asme, 2001. 123(2): p. 134-144. 
14. Longest, P.W. and S. Vinchurkar, Effects of mesh style and grid convergence on particle deposition in bifurcating airway models with comparisons to experimental data. Medical Engineering \& Physics, 2007. 29(3): p. 350-366.

15. Vinchurkar, S. and P.W. Longest, Evaluation of hexahedral, prismatic and hybrid mesh styles for simulating respiratory aerosol dynamics. Computers \& Fluids, 2008. 37(3): p. 317-331.

16. Puso, M.A. and J. Solberg, A stabilized nodally integrated tetrahedral. International Journal for Numerical Methods in Engineering, 2006. 67(6): p. 841-867.

17. Mac Donald, B.J., Practical Stress Analysis with Finite Elements. Glasnevin Publishing, 2007: p. pag 202-203.

18. Viceconti, M., L. Bellingeri, L. Cristofolini, and A. Toni, A comparative study on different methods of automatic mesh generation of human femurs. Medical Engineering \& Physics, 1998. 20(1): p. 1-10.

19. Holzapfel, G.A., G. Sommer, C.T. Gasser, and P. Regitnig, Determination of layerspecific mechanical properties of human coronary arteries with nonatherosclerotic intimal thickening and related constitutive modeling. American Journal of Physiology-Heart and Circulatory Physiology, 2005. 289(5): p. H2048-H2058.

20. Arko, F.R., E.H. Murphy, C.M. Davis, E.D. Johnson, S.T. Smith, and C.K. Zarins, Dynamic geometry and wall thickness of the aortic neck of abdominal aortic aneurysms with intravascular ultrasonography. Journal of Vascular Surgery, 2007. 46(5): p. 891-897.

21. Auricchio, F., Conti, M, De Beule, M, De Santis, G, Verhegghe, B, Carotid artery stenting simulation: From patient-specific images to finite element analysis. medical engineering and physics, 2010.

22. Shepherd, J., Y. Zhang, C. J. Tuttle, C. T. Silva, Quality Improvement and Boolean-like Cutting Operations in Hexahedral Meshes. The 10th ISGG Conference on Numerical Grid Generation. FORTH, Crete, Greece. Sept. 16-20, 2007, 2007.

23. Mortier, P., G.A. Holzapfel, M. De Beule, D. Van Loo, Y. Taeymans, P. Segers, P. Verdonck, and B. Verhegghe, A Novel Simulation Strategy for Stent Insertion and Deployment in Curved Coronary Bifurcations: Comparison of Three Drug-Eluting Stents. Annals of Biomedical Engineering, 2010. 38(1): p. 88-99.

24. Verma, C.S., P.F. Fischer, S.E. Lee, and F. Loth, An all-hex meshing strategy for bifurcation geometries in vascular flow simulation, in Proceedings of the 14th International Meshing Roundtable, B.W. Hanks, Editor. 2005. p. 363-375.

25. Antiga, L. and D.A. Steinman, Robust and objective decomposition and mapping of bifurcating vessels. Ieee Transactions on Medical Imaging, 2004. 23(6): p. 704-713.

26. Murdoch, P., S. Benzley, T. Blacker, and S.A. Mitchell, The spatial twist continuum: A connectivity based method for representing all-hexahedral finite element meshes. Finite Elements in Analysis and Design, 1997. 28(2): p. 137-149. 


\section{APPENDIX}

\section{A.1 Creation of block-structures}

From an STL model, centerlines are computed in VMTK and for each point of the centerline a local triad and a radius are given. The centerlines can be re-sampled because only few points on each centerline are needed for the construction of the blocks. From the points, triads and radii of the centerlines, a series of squares is constructed. Each square is oriented according to the curvature of the vessel (computed with the method of the parallel transport normal) and scaled according to the local radius. By connecting together the squares of each branch and then interconnecting the branches together, the block-structures are obtained. The centerlines describe the vessel as a series of tubular branches but do not take into account the details of the vessel surface. Thus, the vessel surface is generally inside the block-structures in regions where the vessel is nearly tubular and curved with high radius of curvature but may develop outside the block-structures in more complex regions, such as the aortic arch, which contains multiple bifurcations and branches with different radii. In this case, the user needs to displace few points to adapt the block-structures to the surface. The user-intervention in geometrically-complex regions is required because (1) if at some locations the vessel surface remains outside the blockstructures, the projection operation may fail and (2) if the block-structures intersect each others, the final mesh will contain highly distorted elements (Figure a1). Remarkably, the construction of the block-structures does not need the centerline description of the vasculature, and can be performed completely by the user. However, the centerlines allow a quick construction of initial blocks, which can be then fine-tuned to reach the final structures, facilitating and speeding up the mesh generation process. 


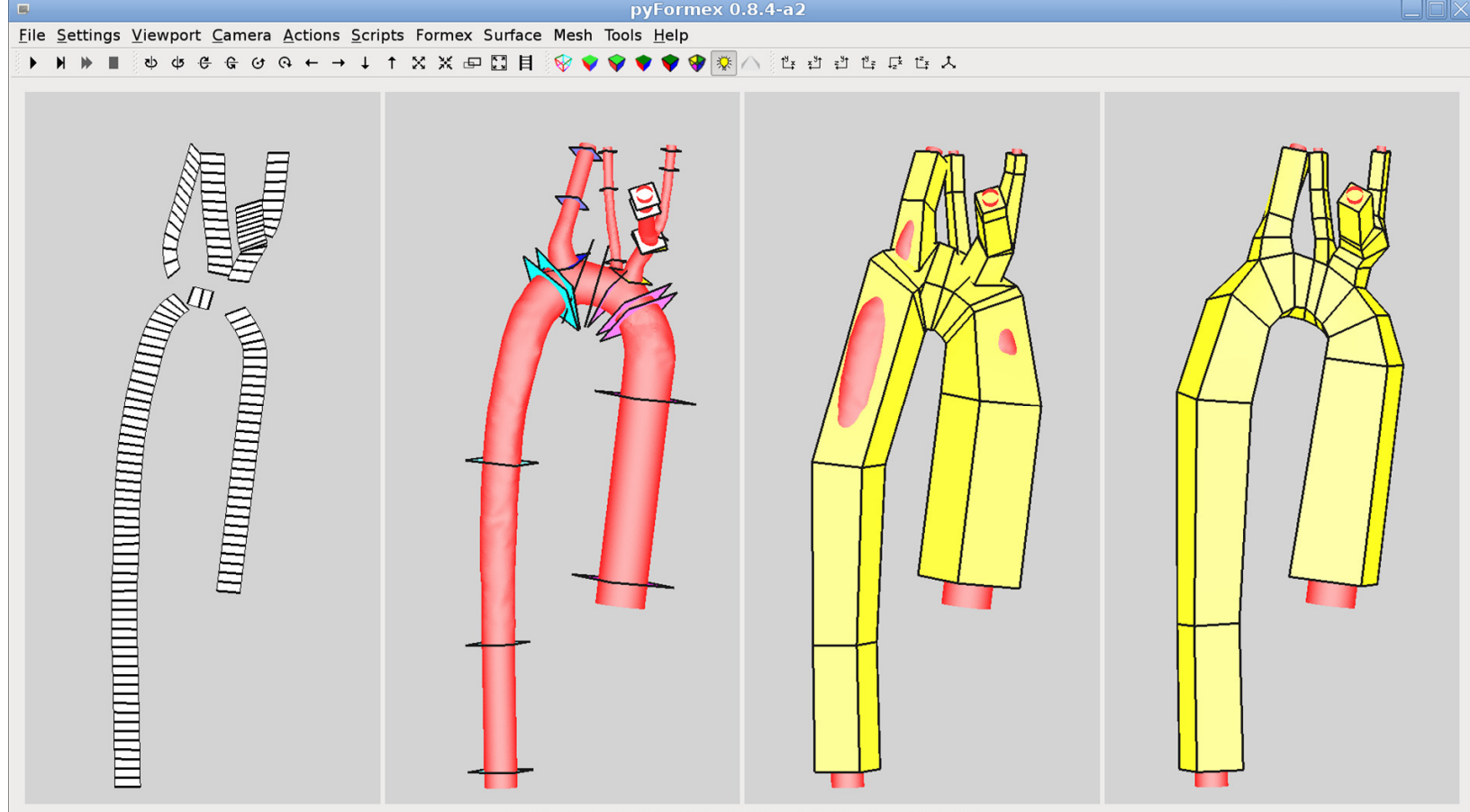

Fig. a1 - Construction of block-structures from centerlines.

The aortic arch is imaged from the back. From left to right: (1) representation of centerlines and triads; (2) squares obtained directly from centerlines, triads and radii; (3) block-structures before the user-intervention, showing regions of vessel surface developing outside the blocks and some blocks intersecting each others; (4) block-structures after user-intervention.

\section{A.2 Remeshing}

The difference between pre-projection and post-projection remeshing can be demonstrated on a perfect spherical surface (Fig. a2 A), discretized into a coarse triangulated model (Fig. a2 B). Such model is meshed using the novel procedure and refined before projection (Fig. a2 C) and after projection (Fig. a2 D). Visual evaluation suggests that the mesh refined before projection (Fig. a2 C) does better resemble the triangulated sphere, inheriting the sharp edges of the triangles (Fig. a2 B), while the mesh refined after projection (Fig. a2 D) does better resemble the original sphere (Fig. a2 A). 


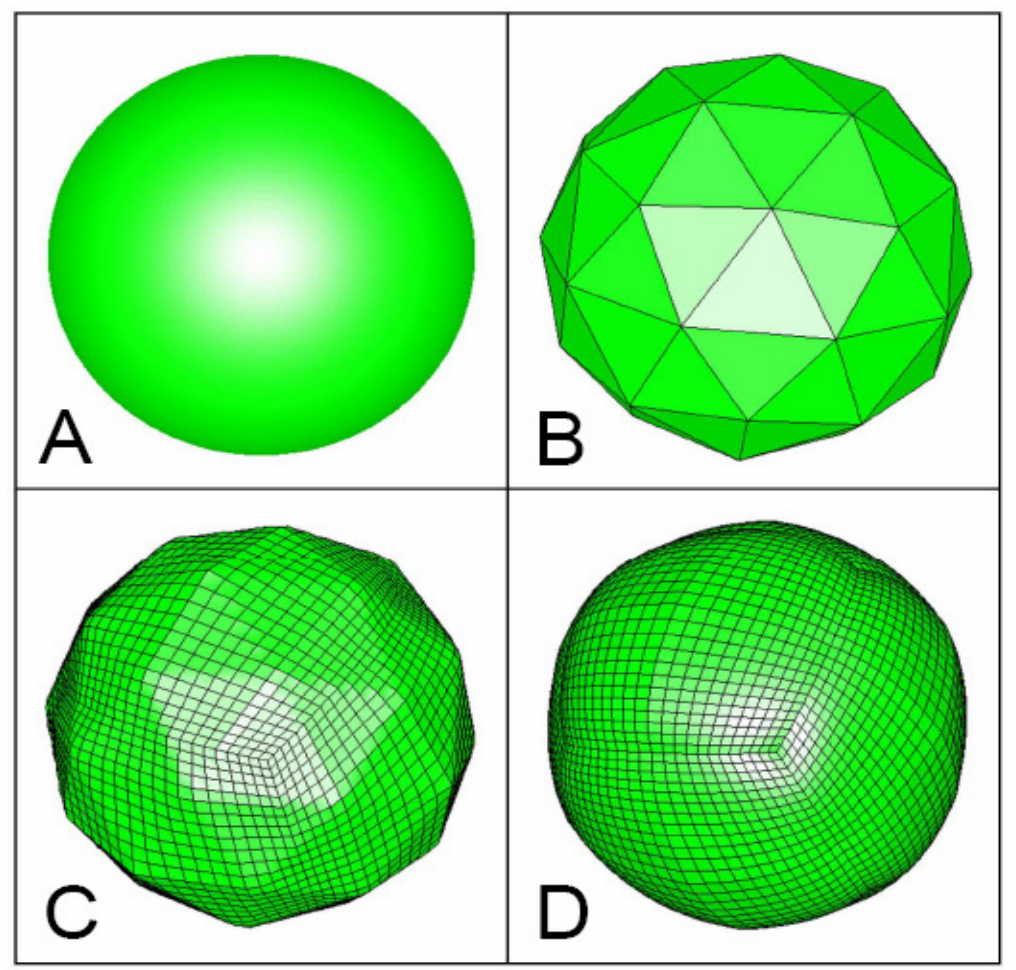

Fig. a2 - Effect of pre-projection and post-projection remeshing.

A sphere (a) is reconstructed as a triangulated surface model of 80 triangles (b). A mesh of 55296 elements (3456 quadrilaterals on the surface) is build inside the sphere volume by refining the block-structures before projection (c, 10370 points projected) and after projection (d, 74 points projected). 